\title{
4D Einstein-Gauss-Bonnet gravity with nonlinear electrodynamics: entropy, energy emission, quasinormal modes and deflection angle
}

\author{
S. I. Kruglov ${ }^{1}$ \\ Department of Physics, University of Toronto, \\ 60 St. Georges St., Toronto, ON M5S 1A\%, Canada \\ Department of Chemical and Physical Sciences, University of Toronto, \\ 3359 Mississauga Road North, Mississauga, Ontario L5L 1C6, Canada
}

\begin{abstract}
The logarithmic correction to Bekenshtein-Hawking entropy in the framework of 4D Einstein-Gauss-Bonnet gravity coupled with nonlinear electrodynamics is obtained. We explore the black hole solution with the spherically symmetric metric. The logarithmic term in the entropy has a structure similar to the entropy correction in the semi-classical Einstein equations which mimics the quantum correction to the area low. The energy emission rate of black holes and energy conditions are studied. Quasinormal modes of black holes are investigated. The gravitational lensing of light around BHs was investigated. We calculated the deflection angle for some model parameters.
\end{abstract}

Keywords: Einstein-Gauss-Bonnet gravity; nonlinear electrodynamics; entropy; energy emission; quasinormal modes; deflection angle

\section{Introduction}

A modification of Einstein General Relativity (GR) by including higher order curvature terms in action is of interest because such terms can mimic quantum corrections. One of the ways is to add the Gauss-Bonnet (GB) term to Einstein-Hilbert action. Such theory, $4 D$ Einstein-Gauss-Bonnet (EGB) gravity in four dimension, recently attracted much attention [1]-[17]). It was shown by Glavan and Lin [1] that $4 D$ EGB theory gives non-trivial dynamics

\footnotetext{
${ }^{1}$ E-mail: serguei.krouglov@utoronto.ca
} 
by the dimension regularization although the GB term is a topological invariant. In [18], [19] the authors obtained a solution of the semi-classical Einstein equations with conformal anomaly that also is a solution in the regularized 4D EGB gravity. Recently, the regularization scheme of Glavan and Lin was debated in [20]-[24]. It is worth noting that the scheme of [25], [26] gives the solution, in the spherically-symmetric metrics, that is a solution in the framework of dimensional regularization [1] (see [27]). Here, we obtain a BH solution in the $4 D$ EGB gravity theory coupled to nonlinear electrodynamics (NED) proposed in [28] making use of the regularization scheme of [1]. This paper is the continuation of the work [29].

The paper is organized as follows. In Sec. 2, we consider BH spherically symmetric solution jn the framework of the $4 D$ EGB gravity. It is shown that at infinity we have the Reissner-Nordström behavior of the charged BH. We obtained the logarithmic correction to Bekenstein-Hawking entropy in Sec. 3. In Sec. 4 we study the $\mathrm{BH}$ energy emission rate. The energy conditions are investigated in Sec. 5. It was shown that WEC, DEC and SEC are satisfied. In Sec. 6 we investigate the $\mathrm{BH}$ quasinormal modes and obtained the corresponding frequencies. We study the light defection angle by the $\mathrm{BH}$ solution in Sec. 7. Section 8 is a conclusion.

\section{The model}

With the help of dimensional regularization of [1] the EGB gravity action in D-dimensions coupled to NED is

$$
I=\int d^{D} x \sqrt{-g}\left[\frac{1}{16 \pi G}\left(R+\frac{\alpha}{D-4} \mathcal{L}_{G B}\right)+\mathcal{L}_{N E D}\right]
$$

with $\alpha$ possessing the dimension of (length) ${ }^{2}$ and the NED Lagrangian, proposed in [28] is given by

$$
\mathcal{L}_{N E D}=-\frac{\mathcal{F}}{1+\sqrt[4]{2 \beta \mathcal{F}}},
$$

where the parameter $\beta(\beta \geq 0)$ has the dimension of (length $)^{4}, \mathcal{F}=(1 / 4) F_{\mu \nu} F^{\mu \nu}=$ $\left(B^{2}-E^{2}\right) / 2, F_{\mu \nu}$ is the strength tensor and the GB Lagrangian is

$$
\mathcal{L}_{G B}=R^{\mu \nu \alpha \beta} R_{\mu \nu \alpha \beta}-4 R^{\mu \nu} R_{\mu \nu}+R^{2} .
$$


The field equation corresponding to action (1) reads

$$
R_{\mu \nu}-\frac{1}{2} g_{\mu \nu} R+\frac{\alpha}{D-4} H_{\mu \nu}=-8 \pi G T_{\mu \nu}
$$

where

$$
H_{\mu \nu}=2\left(R R_{\mu \nu}-2 R_{\mu \alpha} R_{\nu}^{\alpha}-2 R_{\mu \alpha \nu \beta} R^{\alpha \beta}-R_{\mu \alpha \beta \gamma} R_{\nu}^{\alpha \beta \gamma}\right)-\frac{1}{2} \mathcal{L}_{G B} g_{\mu \nu}
$$

It was shown [31] that to have a regular solution as $r \rightarrow 0$ one can consider a magnetic BH. The solution to Eq. (4) in the static and spherically symmetric metric gives the metric function [29]

$$
f(r)=1+\frac{r^{2}}{2 \alpha}\left(1-\sqrt{1+\frac{8 M \alpha G}{r^{3}}+\frac{4 \alpha q_{m}^{3 / 2} G}{\beta^{1 / 4} r^{3}} \ln \left(\frac{r}{r+\sqrt[4]{\beta q_{m}^{2}}}\right)}\right)
$$

where $q_{m}$ is a magnetic charge. With the dimensionless variable $x=r / \sqrt[4]{\beta q_{m}^{2}}$, Eq. (6) is rewritten as

$$
f(x)=1+c x^{2}-c \sqrt{x^{4}+x\left(a+b \ln \left(\frac{x}{x+1}\right)\right)},
$$

where we use the dimensionless parameters

$$
a=\frac{8 M \alpha G}{\beta^{3 / 4} q_{m}^{3 / 2}}, \quad b=\frac{4 \alpha G}{\beta}, \quad c=\frac{\sqrt{\beta} q_{m}}{2 \alpha},
$$

where $M$ is the $\mathrm{BH}$ mass and we choose the branch with the sign minus in Eqs. (6) and (7) to have the stable BH [30]. The asymptotic of the metric function $f(r)$ (6) as $r \rightarrow \infty$ is given by

$$
f(r)=1-\frac{2 M G}{r}+\frac{G q_{m}^{2}}{r^{2}}+\mathcal{O}\left(r^{-3}\right) \quad r \rightarrow \infty .
$$

This equation shows the Reissner-Nordström behavior of the charged BH at infinity. 


\section{Thermodynamics and BH entropy}

The Hawking temperature in our model is given by [29]

$$
T_{H}\left(x_{+}\right)=\frac{\left(2 c x_{+}^{2}-1\right)\left(1+x_{+}\right)-b c^{2} x_{+}}{8 \pi \sqrt[4]{\beta q_{m}^{2}} x_{+}\left(1+x_{+}\right)\left(1+c x_{+}^{2}\right)},
$$

and the $\mathrm{BH}$ gravitational mass reads

$$
M\left(x_{+}\right)=\frac{\beta^{3 / 4} q_{m}^{3 / 2}}{8 \alpha G}\left(\frac{1+2 c x_{+}^{2}}{c^{2} x_{+}}-b \ln \left(\frac{x_{+}}{x_{+}+1}\right)\right) .
$$

According to the first law of $\mathrm{BH}$ thermodynamics the entropy at the constant charge is

$$
S=\int \frac{d M\left(x_{+}\right)}{T_{H}\left(x_{+}\right)}=\int \frac{1}{T_{H}\left(x_{+}\right)} \frac{\partial M\left(x_{+}\right)}{\partial x_{+}} d x_{+} .
$$

Making use of Eqs. (9), (10) and (11) we obtain the entropy

$$
S=\frac{4 \pi \alpha}{G} \int \frac{1+c x_{+}^{2}}{x_{+}} d x_{+}=\frac{\pi r_{+}^{2}}{G}+\frac{4 \pi \alpha}{G} \ln \left(\frac{r_{+}}{\sqrt[4]{\beta q_{m}^{2}}}\right)
$$

We put here the integration constant to be zero. Equation (12) shows that the entropy in our model besides the Bekenstein-Hawking area low includes the logarithmic correction. It should be noted that similar logarithmic term is also present in GR with a conformal anomaly, loop quantum gravity and string theory [18], [19], [32]. Thus, the logarithmic correction obtained mimics the quantum correction which is due to the GB Lagrangian. When the coupling constant of GB term $\alpha$ vanishes we come to the Bekenstein-Hawking entropy. For the massive BHs with the large event horizon radius $x_{+}$the leading term to the entropy is the Bekenstein-Hawking area law. But for small $x_{+}$(for the light BHs) the quantum corrections take place and the logarithmic term is important. It is worth noting that at some values of parameters $\alpha, \beta, q_{m}$ and $r_{+}$the entropy is zero. The event horizon radius, when the entropy vanishes, is the solution of equation $(S=0)$ $r_{+}^{4} \exp \left(r_{+}^{2} / \alpha\right)=\beta q_{m}^{2}$ which is $r_{0}=\sqrt{2 \alpha W_{0}\left(\sqrt{\beta} q_{m} /(2 \alpha)\right)}$, where $W_{0}(x)$ is the Lambert function. It is interesting that at $r_{+}<r_{0}$ the entropy becomes negative which can indicate a new type of instability. Probably, for such values of $r_{0}$ the $\mathrm{BH}$ does not exist. It is worth noting that the negative entropy of BHs was discussed in [33]. 


\section{The energy emission rate}

The particle emission rate of the $\mathrm{BH}$ depends on the model parameters $\alpha$, $\beta, q_{m}$ and $M$. The $\mathrm{BH}$ shadow can be connected with the high energy absorption cross section $\sigma$ for the observer located at infinity [34]-[36] (see also [37]). The absorption cross-section, at very high energies, oscillates around an approximate value of the photon sphere $\sigma \approx \pi r_{s}^{2}$, where $x_{s}$ is the $\mathrm{BH}$ shadow radius. The energy emission rate in the high energy is given by

$$
\frac{d^{2} E(\omega)}{d t d \omega}=\frac{2 \pi^{3} \omega^{3} r_{s}^{2}}{\exp \left(\omega / T_{H}\left(r_{+}\right)\right)-1},
$$

where $\omega$ denotes the emission frequency and $T_{H}$ is the Hawking temperature. With the help of the dimensionless variable $x=r / \sqrt[4]{\beta q_{m}^{2}}, \bar{T}_{H}\left(x_{+}\right)=$ $\sqrt[4]{\beta q_{m}^{2}} T_{H}\left(x_{+}\right)$, and Eq. (13), we obtain

$$
\beta^{1 / 4} \sqrt{q_{m}} \frac{d^{2} E(\omega)}{d t d \omega}=\frac{2 \pi^{3} \varpi^{3} x_{s}^{2}}{\exp \left(\varpi / \bar{T}_{H}\left(x_{+}\right)\right)-1},
$$

where the Hawking temperature is given by Eq. (9) and $\varpi=\beta^{1 / 4} \sqrt{q_{m}} \omega$. Making use of the data given in Table 1 (see [29]) we depicted the plot of the emission rate in Fig. 1 for $c=1, a=5$ and $b=1.5,2,2.5$. According

Table 1: The event horizon, photon sphere and shadow dimensionless radii for $\mathrm{a}=5, \mathrm{c}=1$

\begin{tabular}{cccccccccccc}
\hline$b$ & 0.5 & 0.9 & 1.5 & 1.7 & 1.8 & 2 & 2.2 & 2.3 & 2.4 & 2.5 & 2.6 \\
\hline$x_{+}$ & 2.18 & 2.08 & 1.93 & 1.87 & 1.84 & 1.77 & 1.69 & 1.65 & 1.61 & 1.56 & 1.51 \\
\hline$x_{p}$ & 3.42 & 3.31 & 3.12 & 3.05 & 3.01 & 2.94 & 2.86 & 2.82 & 2.77 & 2.73 & 2.68 \\
\hline$x_{s}$ & 6.16 & 6.02 & 5.78 & 5.70 & 5.65 & 5.56 & 5.47 & 5.42 & 5.37 & 5.32 & 5.26 \\
\hline
\end{tabular}

to Fig. 1 there is a peak of the energy emission rate for the $\mathrm{BH}$ depending on model parameters. When the parameter $b$ increases, the maximum of the peak decreases and possesses the low frequency. Thus, the $\mathrm{BH}$ has a bigger lifetime at a bigger parameter $b$. One can investigate the dependance of the energy emission rate on parameters, putting numerical values for $\alpha, \beta, q_{m}$ and $M$. 


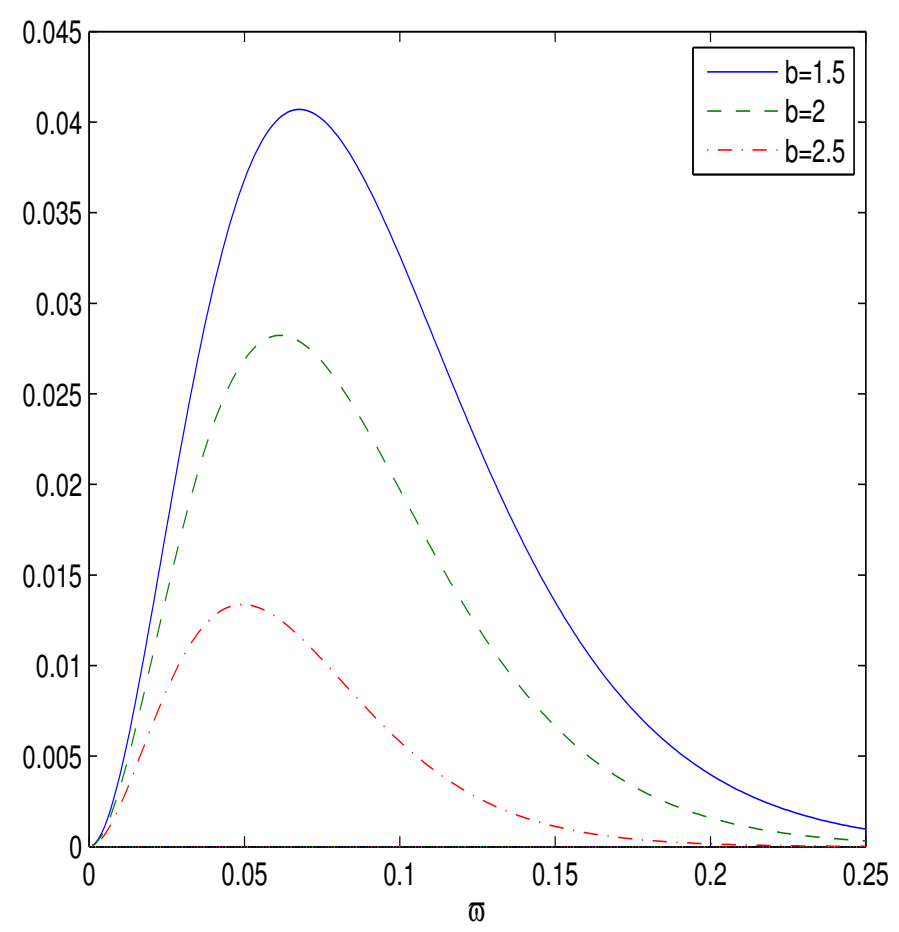

Figure 1: The plot of the function $\beta^{1 / 4} \sqrt{q_{m}} \frac{d^{2} E(\omega)}{d t d \omega}$ vs. $\varpi$ for $b=1.5,2,2.5$, $a=5, c=1$.

\section{The energy conditions}

The symmetrical energy-momentum tensor with the spherically symmetry leads to $T_{t}{ }^{t}=T_{r}{ }^{r}$ and the radial pressure is given by $p_{r}=-T_{r}{ }^{r}=-\rho$. The tangential pressure is defined as $p_{\perp}=-T_{\vartheta}{ }^{\vartheta}=-T_{\phi}{ }^{\phi}$ so that [38]

$$
p_{\perp}=-\rho-\frac{r}{2} \rho^{\prime}(r)
$$

where the prime means the derivative with respect to the argument. The Weak Energy Condition (WEC) is satisfied when $\rho \geq 0$ and $\rho+p_{k} \geq 0$ $(\mathrm{k}=1,2,3)$ [39]. This guarantees that the energy density is non-negative as measured by any local observer. The magnetic energy density in our model 
is $[29]$

$$
\rho=T_{t}^{t}=\frac{B^{2}}{2\left(\beta^{1 / 4} \sqrt{B}+1\right)}=\frac{q_{m}^{2}}{2 r^{3}\left(r+\sqrt{q_{m}} \beta^{1 / 4}\right)},
$$

where $q_{m}$ is a magnetic charge, $\mathcal{F}=q_{m}^{2} /\left(2 r^{4}\right)$. In accordance with Eq. (16) $\rho \geq 0$. Making use of Eq. (16) one finds

$$
\rho^{\prime}(r)=-\frac{q_{m}^{2}\left(4 r+3 \beta^{1 / 4} \sqrt{q_{m}}\right)}{2 r^{4}\left(r+\beta^{1 / 4} \sqrt{q_{m}}\right)^{2}} \leq 0 .
$$

As a result, we have $\rho \geq 0, \rho+p_{r}=0, \rho+p_{\perp} \geq 0$ and WEC holds. The Dominant Energy Condition (DEC) takes place when [39] $\rho \geq o, \rho+p_{k} \geq 0$, $\rho-p_{k} \geq 0$. These conditions include WEC and we have to verify the condition $\rho-p_{\perp} \geq 0$. From Eqs. (15), (16) and (17) we obtain

$$
\rho-p_{\perp}=\frac{q_{m}^{5 / 2} \beta^{1 / 4}}{4 r^{3}\left(r+\sqrt{q_{m}} \beta^{1 / 4}\right)^{2}} \geq 0 .
$$

Thus, DEC is satisfied. As a result, the sound speed cannot exceed the speed of light. The Strong Energy Condition (SEC) requires the condition $\rho+\sum_{k=1}^{3} p_{k} \geq 0$ [39]. With the help of Eqs. (15), (16) and (17) we obtain

$$
\rho+\sum_{k=1}^{3} p_{k}=2 p_{\perp}=\frac{q_{m}^{2}\left(2 r+\sqrt{q_{m}} \beta^{1 / 4}\right)}{2 r^{3}\left(r+\sqrt{q_{m}} \beta^{1 / 4}\right)^{2}} \geq 0,
$$

and SEC is satisfied.

\section{Quasinormal Modes}

Quasinormal modes (QNMs) are characterised by complex frequencies $\omega$ which give an information about the stability of BHs under small perturbations and they do not depend on the initial conditions. The outgoing boundary condition is imposed at infinity and the ingoing boundary condition at the event horizon. If $\operatorname{Im} \omega>0$ the mode is unstable, otherwise it is stable. It was shown that Re $\omega$ in the eikonal limit is linked with the radius of the BH shadow [40], [41]. The perturbations by a scalar massless field around BHs are characterized by the effective potential barrier

$$
V(r)=f(r)\left(\frac{f^{\prime}(r)}{r}+\frac{l(l+1)}{r^{2}}\right)
$$


where $l$ being the multipole number $0,1,2 \ldots$ Equation (20) can be represented in the terms of dimensionless variable $x=r / \sqrt[4]{\beta q_{m}^{2}}$ as

$$
V(x) \sqrt{\beta} q_{m}=f(x)\left(\frac{f^{\prime}(x)}{x}+\frac{l(l+1)}{x^{2}}\right) .
$$

The effective potential is plotted in Fig. 2 for $a=5, b=2, c=1$ and $l=1,2,3$ and for $a=5, c=1, l=1$ and $b=1,2,3$. According to Fig.

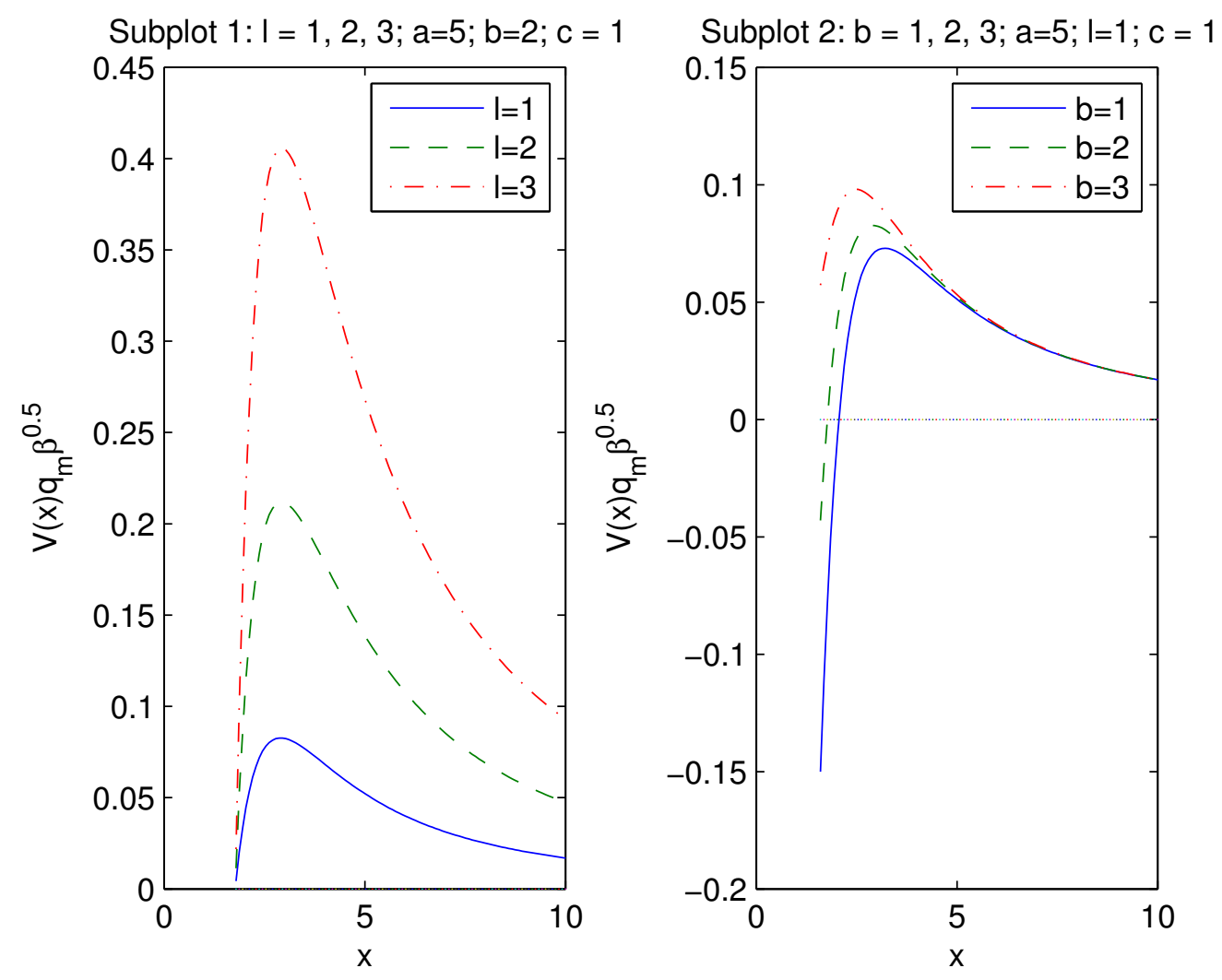

Figure 2: The plot of the function $V(x) \sqrt{\beta} q_{m}$ for $a=5, c=1$.

2, Subplot 1, shows that the effective potentials represent a potential barrier with a maximum. The height of the potential increases when the $l$ increases. In accordance with Fig.2, Subplot 2, the height of the potential increases if the parameter $b$ increases. The real and imaginary parts of quasinormal 
frequencies are given by [40], [41]

$$
\operatorname{Re} \omega=\frac{l}{r_{s}}=\frac{l \sqrt{f\left(r_{p}\right)}}{r_{p}}, \quad \operatorname{Im} \omega=-\frac{2 n+1}{2 \sqrt{2} r_{s}} \sqrt{2 f\left(r_{p}\right)-r_{p}^{2} f^{\prime \prime}\left(r_{p}\right)},
$$

where $r_{s}$ is the $\mathrm{BH}$ shadow radius (the impact parameter), $r_{p}$ is the radius of the $\mathrm{BH}$ photon sphere, $n=0,1,2, \ldots$ is the overtone number. The real and imaginary parts of the frequencies versus the parameter $b$ at $a=5, c=1$, $n=1, l=5$ are given in Table 2 . The imaginary parts of the frequencies

Table 2: The real and the imaginary parts of the frequencies vs the parameter $b$ at $n=1, l=5, a=5, c=1$

\begin{tabular}{cccccccc}
\hline$b$ & 1.5 & 1.7 & 2 & 2.2 & 2.4 & 2.5 & 2.6 \\
\hline$\sqrt[4]{\beta q_{m}^{2}} \operatorname{Re} \omega$ & 0.865 & 0.877 & 0.899 & 0.914 & 0.931 & 0.940 & 0.951 \\
\hline$-\sqrt[4]{\beta q_{m}^{2}} \operatorname{Im} \omega$ & 0.2212 & 0.2208 & 0.2202 & 0.2191 & 0.2170 & 0.2163 & 0.2149 \\
\hline
\end{tabular}

in Table 2 are negative, and therefore, the modes are stable and the real part represents the frequency of oscillations. According to Table 2 when the parameter $b$ increases the real part of the reduced frequency $\sqrt[4]{\beta q_{m}^{2}} \operatorname{Re} \omega$ increases, but absolute value of the imaginary part of the reduced frequency $\left|\sqrt[4]{\beta q_{m}^{2}} \operatorname{Im} \omega\right|$ decreases. In other words, increasing the parameter $b$ the scalar perturbations oscillate with greater frequency and decay slowly. To study the dependance of frequencies on parameters $\alpha, \beta, M, q_{m}$ one has to put numerical numbers for these parameters in Eq. (22).

\section{Deflection angle}

Let us study the light deflection angle by the BH solution (6). We can determine the total deflection angle $\Delta \varphi$ by the formula [42] (see also [43])

$$
\Delta \varphi=2 \int_{r_{p}}^{\infty} \frac{d r}{r \sqrt{\frac{r^{2} f\left(r_{p}\right)}{r_{p}^{2}}-f(r)}}-\pi
$$

where $r_{p}$ is the photon sphere radius. Taking into account that $r_{p} / \sqrt{f\left(r_{p}\right)}=$ $r_{s}$ is the shadow sphere radius $\left(r_{s}=\xi\right.$ is the impact parameter) one can 
represent Eq. (23) in terms of the dimensionless variable as

$$
\Delta \varphi=2 \int_{x_{p}}^{\infty} \frac{d x}{x \sqrt{\frac{x^{2}}{x_{s}^{2}}-f(x)}}-\pi
$$

Making use of Table 1 we obtain the deflection angles from Eq. (24) represented in Table 3. According to Table 3 when the parameter $b$ increasing,

Table 3: The deflection angles for $\mathrm{a}=5, \mathrm{c}=1$

\begin{tabular}{cccccccccccc}
\hline$b$ & 0.5 & 0.9 & 1.5 & 1.7 & 1.8 & 2 & 2.2 & 2.3 & 2.4 & 2.5 & 2.6 \\
\hline$\Delta \varphi$ & 4.12 & 3.58 & 3.1 & 3.02 & 2.96 & 2.86 & 2.81 & 2.77 & 2.76 & 2.73 & 2.69 \\
\hline
\end{tabular}

with fixed $a$ and $c$, the deflection angle is decreased. One can also study the dependence of the deflection angle on parameters $\beta, \alpha, q_{m}$ and $M$ by taking the numerical values for these parameters in Eq. (6), finding the solution for $r_{p}: 2 f\left(r_{p}\right)-r_{p} f^{\prime}\left(r_{p}\right)=0\left(r_{s}=r_{p} / \sqrt{f\left(r_{p}\right)}\right)$, and calculating the integral (23).

\section{Conclusion}

We use the exact spherically symmetric and magnetically charged $\mathrm{BH}$ solution in 4D EGB gravity coupled to NED obtained in [29] for further investigations. It is shown that the Reissner-Nordström behavior of the charged $\mathrm{BH}$ at infinity. The logarithmic correction to the Bekenstein-Hawking entropy mimicking a quantum correction was obtained. For the light BHs the logarithmic correction is important while for massive BHs (for big event horizon radius) such correction is small. We shown that the $\mathrm{BH}$ energy emission rate decreases with increasing the model parameter $b$ and the $\mathrm{BH}$ has a bigger lifetime. It was demonstrated that WEC, DEC and SEC are satisfied. We investigated the $\mathrm{BH}$ quasinormal modes and it was demonstrated that increasing the parameter $b$ the scalar perturbations oscillate with greater frequency and decay slowly. The deflection angle $\Delta \varphi$ for some parameters was calculated. It was shown that $\Delta \varphi$ is decreased if the parameter $b$ increasing at fixed $a$ and $c$. Thus, we studied the influence of the BH solution in $4 \mathrm{D}$ EGB gravity on optical behaviour of the BHs. 


\section{References}

[1] D. Glavan and C. Lin, Phys. Rev. Lett. 124, 081301 (2020).

[2] P. G. S. Fernandes, Phys. Lett. B 805135468 (2020).

[3] R. A. Konoplya and A. Zhidenko, Phys. Rev. D 101, 084038 (2020).

[4] K. Jusufi, Ann. Phys. 421, 168285 (2020).

[5] S. G. Ghosh, D. V. Singh, R. Kumar, and S. D. Maharaj, Ann. Phys. 424, 168347 (2021).

[6] S. G. Ghosh and S. D. Maharaj, Phys. Dark Univ. 30, 100687 (2020).

[7] R. Kumar and S. G. Ghosh, JCAP 07, 053 (2020).

[8] X. H. Jin, Y. X. Gao, and D. J. Liu, Int. J. Mod. Phys. D 29, 2050065 (2020).

[9] K. Jusufi, A. Banerjee, and S. G. Ghosh, Eur. Phys. J. C 80, 698 (2020).

[10] M. Guo and P. Li, Eur. Phys. J. C 80, 588 (2020).

[11] C. Zhang, S. Zhang, P. Li, and M. Guo, JHEP 08, 105 (2020).

[12] C-Y. Zhang, P-C. Li, and M. Guo, Eur. Phys. J. C 80, 874 (2020).

[13] S. Odintsov, V. Oikonomou, and F. Fronimos, Nucl. Phys. B 958, 115135 (2020).

[14] W. Ai, Commun. Theor. Phys. 72, 095402 (2020).

[15] P. G. Fernandes, P. Carrilho, T. Clifton, and D. J. Mulryne, Phys. Rev. D 102, $024025(2020)$.

[16] B. E. Panah, K. Jafarzade and S. H. Hendi, Nucl. Phys. B 961, 115269 (2020).

[17] R. A. Hennigar, D. Kubiznak, R. B. Mann, and C. Pollack, JHEP 2020, 27 (2020).

[18] R. G. Cai, L. M. Cao, and N. Ohta, JHEP 1004, 082 (2010). 
[19] R.-G. Cai, Phys. Lett. B 733, 183 (2014).

[20] M. Gurses, T. C. Sisman, and B. Tekin, Phys. Rev. Lett. 125, 149001 (2020).

[21] M. Gurses, T. C. Sisman, and B. Tekin, Eur. Phys. J. C 80, 647 (2020).

[22] S. Mahapatra, Eur. Phys. J. C 80, 992 (2020).

[23] J. Arrechea, A. Delhom, and A. Jiménez-Cano, Chin. Phys. C 45, 013107 (2021).

[24] M, Hohmann, C. Pfeifer, N. Voicu, Eur. Phys. J. Plus 136, 180 (2021).

[25] K. Aoki, M. A. Gorji, and S. Mukohyama, Phys. Lett. B 810, 135843 (2020).

[26] K. Aoki, M. A. Gorji, and S. Mukohyama, JCAP 2009, 014 (2020).

[27] K.e Jafarzade, M. K. Zangeneh, F. S.N. Lobo, Shadow, deflection angle and quasinormal modes of Born-Infeld charged black holes, 2010.05755 [gr-qc].

[28] S. I. Kruglov, Ann. Phys. (Berlin) 529, 1700073 (2017).

[29] S. I. Kruglov, Symmetry 13, 204 (2021).

[30] D. G. Boulware and S. Deser, Phys. Rev. Lett. 55, 2656 (1985).

[31] K. A. Bronnikov, Phys. Rev. D 63, 044005 (2001).

[32] G. Cognola, R. Myrzakulov, L. Sebastiani, and S. Zerbini, Phys. Rev. D 88, 024006 (2013),

[33] M. Cvetic, S. Nojiri, and S. D. Odintsov, Nucl. Phys. B 628, 295 (2002).

[34] B. Mashhoon, Phys. Rev. D 7, 2807 (1973).

[35] C.W. Misner, K. S. Thorne, and J. A. Wheeler, Gravitation (Freeman, San Francisco, 1973).

[36] Y. Decanini, G. Esposito-Farese, and A. Folacci, Phys. Rev. D 83, 044032 (2011) 
[37] S. W. Wei and Y. X. Liu, JCAP 11, 063 (2013).

[38] I. Dymnikova, Class. Quant. Grav. 21, 4417 (2004).

[39] S. W. Hawking and G. F. R. Ellis, The large scale structure of spacetime, Canbridge Univ. Press (1973).

[40] K. Jusufi, Phys. Rev. D 101, 084055 (2020).

[41] K. Jusufi, Phys. Rev. D 101, 124063 (2020).

[42] S. Weinberg, Gravitation and Cosmology: Principles and Applications of the General Theory of Relativity, Wiley, New York (1972).

[43] P. Kocherlakota and L. Rezzolla, Phys. Rev. D 102, 064058 (2020). 